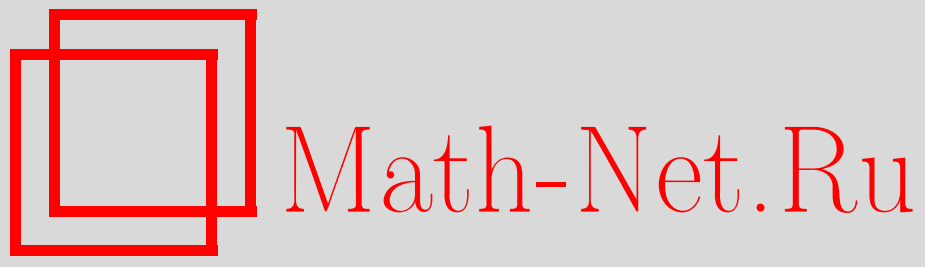

С. В. Асташкин, Кратные ряды Радемахера в симметричных пространствах, Функи. анализ и его прил., 1999, том 33, выпуск 2, 73-76

DOI: https://doi.org/10.4213/faa356

Использование Общероссийского математического портала MathNet.Ru подразумевает, что вы прочитали и согласны с пользовательским соглашением http://www . mathnet.ru/rus/agreement

Параметры загрузки:

IP : 3.80 .181 .102

26 апреля 2023 г., 14:56:43

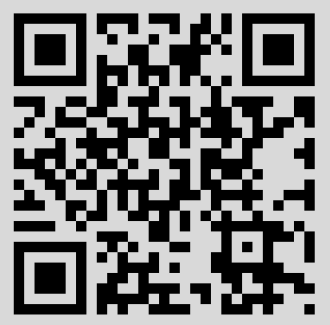


4. Литвинов Г. Л. В кн.: Труды семинара по векторному и тензорному анализу, т. 19, 263-272 (1979); англ. вариант: Selecta Matematica Sovietica, 8, No. 3, 203-212 (1989). 5. Акбаров С. С. Матем. заметки, 57, № 3, 463-466 (1995). 6. Акбаров С. С. Функц. анализ и его прил., 29, вып. 4, 68-72 (1995). 7. Jarchow H. Locally convex spaces. B. G. Teubner, Stuttgart, 1981. 8. MacLane S. Categories for working mathematician. Springer, N. Y., 1971. 9. Артамонов В. А., Салий В. Н., Скорняков Л. А., Шеврин Л. Н., Шульгейфер Е. Г. Общая алгебра. Наука, М., 1991. 10. Szankowski A. Acta Math., 147, 89-108 (1981). 11. Шефер X. Топологические векторные пространства. Мир, М., 1971. 12. Акбаров С. С. Известия РАН. 59, № 1, 3-48 (1995).

Московский государственный

технический университет им. Н. Э. Баумана

Поступило в редакцию e-mail: serge@mx.bmstu.ru

9 декабря 1997 г.

УДК 517.982.27

\section{Кратные ряды Радемахера в симметричных пространствах}

(c) 1999. С. В. Асташкин

Пусть

$$
r_{k}(t)=\operatorname{sign} \sin 2^{k-1} \pi t \quad(k=1,2, \ldots)
$$

- система функций Радемахера на $I=[0,1]$. Рассмотрим множество функций $x(t)$, представимых в виде

$$
x(t)=\sum_{i \neq j} a_{i, j} r_{i}(t) r_{j}(t) \quad(t \in I) .
$$

Как и в случае рядов по системе Радемахера (см., например, [1, с. 148-150]), ряд (1) сходится почти всюду на $I$ (т. е. существует предел его прямоугольных частичных сумм) тогда и только тогда, когда последовательность коэффициентов $a=\left(a_{i, j}\right)_{i \neq j}$ принадлежит $l_{2}$.

Ортонормированная на $I$ система $\left\{r_{i} r_{j}\right\}_{i \neq j}$, в отличие от системы Радемахера, состоит из функций, не являющихся независимыми. Тем не менее ее свойства во многих отношениях напоминают свойства семейств независимых и равномерно ограниченных функций. Так, например, условие $\sum_{i \neq j} a_{i, j}^{2}<$ $\infty$ влечет за собой суммируемость функции $\exp (\alpha|x(t)|)$ для любого $\alpha>0$ $[2$, p. 105]. В то же время есть и существенные отличия. Например, кратная система $\left\{r_{i} r_{j}\right\}_{i \neq j}$ не является системой Сидона [3]. Для более полного изучения ее свойств (в частности, вопроса о возможности усиления утверждения об экспоненциальной суммируемости) целесообразно перейти к общим симметричным пространствам.

Напомним, что банахово пространство $X$ измеримых по Лебегу на $I$ функций $x=x(t)$ называется симметричным пространством (СП), если из неравенства $x^{*}(t) \leqslant y^{*}(t)\left(z^{*}(t)\right.$ - невозрастающая перестановка функции $|z(u)|$ $[4$, c. 83]) и того, что $y \in X$, следует, что $x \in X$ и $\|x\| \leqslant\|y\|$.

Кроме пространств $L_{p}(1 \leqslant p \leqslant \infty)$, важными примерами СП являются пространства Орлича и Марцинкевича. 
Если $S(t) \geqslant 0$ - выпуклая и непрерывная функция на $[0, \infty), S(0)=0$, то пространство Орлича $L_{S}$ состоит из всех $x=x(t)$, для которых

$$
\|x\|_{S}=\inf \left\{u>0: \int_{0}^{1} S\left(\frac{|x(t)|}{u}\right) d t \leqslant 1\right\}<\infty .
$$

Если $\varphi(t) \geqslant 0-$ вогнутая возрастающая функция на $(0,1]$, то пространство Марцинкевича $M(\varphi)$ состоит из всех $x=x(t)$, для которых

$$
\|x\|_{M(\varphi)}=\sup \left\{\frac{1}{\varphi(t)} \int_{0}^{t} x^{*}(s) d s: 0<t \leqslant 1\right\}<\infty .
$$

1. Суммируемость кратных рядов Радемахера. Обозначим через $H$ замыкание пространства $L_{\infty}$ в пространстве Орлича $L_{M}$, где $M(t)=e^{t}-1$.

Теорема 1. Система $\left\{r_{i} r_{j}\right\}_{i \neq j}$ в СП $X$ эквивалентна каноническому базису в $l_{2}$, m.e.

$$
\left\|\sum_{i \neq j} a_{i, j} r_{i} r_{j}\right\|_{X} \asymp\left(\sum_{i \neq j} a_{i, j}^{2}\right)^{1 / 2}
$$

(существует двусторонняя оценка с константами, зависящими только от $X)$, тогда и только тогда, когда $X \supset H$.

ЗАмечАнИЕ 1. Теорема 1 показывает, в частности, неулучшаемость сформулированного в начале работы утверждения об экспоненциальной суммируемости кратных рядов Радемахера.

ЗАмечание 2. Для простых рядов Радемахера справедлив аналогичный результат с заменой $H$ на $G$ - замыкание пространства $L_{\infty}$ в пространстве Орлича $L_{N}, N(t)=e^{t^{2}}-1[5]$.

2. Дополняемость подпространства, порожденного кратной системой Радемахера. Напомним, что норма в СП $X$ называется порядково полунепрерывной, если из того, что $x_{n}=x_{n}(t) \geqslant 0, x_{n}(t) \uparrow x(t)$ при $n \rightarrow \infty$ почти всюду на $I$ и $x \in X$, следует, что $\left\|x_{n}\right\|_{X} \rightarrow\|x\|_{X}$.

Обозначим через $\mathscr{R}(X)$ подпространство СП $X$, состоящее из всех функций вида (1), таких, что $x \in X$.

ТЕОРема 2. Пусть $X$ есть СП с порядково полунепрерьвной нормой. Подпространство $\mathscr{R}(X)$ дополняемо в $X$ тогда и только тогда, когда $H \subset$ $X \subset H^{*}$.

ЗАмЕчАНИЕ 3 . Из теории пространств Орлича [6] следует, что $H^{*}=L_{\widetilde{M}}$, где $\widetilde{M}(u) u^{-1} \ln ^{-1} u \rightarrow 1$ при $u \rightarrow+\infty$.

ЗАМЕЧАНИЕ 4 . Как известно, подпространство, порожденное простой системой Радемахера, дополняемо в СП $X$ тогда и только тогда, когда $G \subset X \subset$ $G^{*}$, где $G^{*}=L_{\widetilde{N}}$ и $\widetilde{N}(t) u^{-1} \ln ^{-1 / 2} u \rightarrow 1$ при $u \rightarrow+\infty$ (см. [7] или $[8,2 . b .4]$ ).

3. Кратная система Радемахера в CП, «близких» к $L_{\infty}$. «Вблизи» пространства $L_{\infty}$ и, в частности, в нем самом поведение кратной системы Радемахера становится гораздо более сложным. 
Для произвольных $n \in \mathbb{N}$ и $\theta=\left\{\theta_{i, j}\right\}_{i, j=1}^{n}, \theta_{i, j}= \pm 1$, введем величину

$$
\varphi_{n}(\theta)=\left\|\sum_{i, j=1}^{n} \theta_{i, j} r_{i} r_{j}\right\|_{\infty}
$$

$\left(\|\cdot\|\right.$ - норма в пространстве $\left.L_{\infty}\right)$.

Из определения функций Радемахера следует, что $\sup _{\theta} \varphi_{n}(\theta)$ достигается при $\theta_{i, j}=1(i, j=1, \ldots, n)$ и равен $n^{2}$.

ТЕОРема 3. С константами, не зависящими от $n \in \mathbb{N}$,

$$
\inf _{\theta} \varphi_{n}(\theta) \asymp 2^{-n^{2}} \sum_{\theta} \varphi_{n}(\theta) \asymp n^{3 / 2} .
$$

ЗАмЕЧАНИЕ 5. Из теоремы 3 , в частности, следует, что система $\left\{r_{i} r_{j}\right\}_{i \neq j}$ не является системой Сидона на $I$. Ранее это утверждение было доказано в [3].

Базисная последовательность $\left\{x_{n}\right\}_{n=1}^{\infty}$ банахова пространства $X$ называется безусловной, если из сходимости ряда $\sum_{n=1}^{\infty} a_{n} x_{n}\left(a_{n} \in \mathbb{R}\right)$ в $X$ следует сходимость в этом пространстве ряда $\sum_{n=1}^{\infty} \theta_{n} a_{n} x_{n}$ для произвольных знаков $\theta_{n}= \pm 1(n=1,2, \ldots)$.

Ввиду теоремы 1 кратная система Радемахера не только безусловна, но и симметрична в СП $X$, если $X \supset H$. Совершенно иначе обстоит дело в пространствах, «близких» к $L_{\infty}$. Рассмотрим подход, позволяющий оценить степень небезусловности кратной системы в этом случае.

Для любой расстановки знаков $\theta=\left\{\theta_{i, j}\right\}_{i, j=1}^{\infty}, \theta_{i, j}= \pm 1$, на $\mathscr{R}\left(L_{\infty}\right)$ определим оператор

где

$$
T_{\theta} x(t)=\sum_{i \neq j} \theta_{i, j} a_{i, j} r_{i}(t) r_{j}(t)
$$

$$
x(t)=\sum_{i \neq j} a_{i, j} r_{i}(t) r_{j}(t) \in L_{\infty}, \quad a=\left(a_{i, j}\right)_{i \neq j} \in l_{2}
$$

ТЕорема 4. Для произвольного $0<\varepsilon<1 / 2$ существует расстановка знаков $\theta=\left\{\theta_{i, j}\right\}$, для которой

$$
T_{\theta}: \mathscr{R}\left(L_{\infty}\right) \not \rightarrow M\left(\varphi_{\varepsilon}\right)
$$

где $M\left(\varphi_{\varepsilon}\right)$ - пространство Мариинкевича, построенное по функиии $\varphi_{\varepsilon}(t)=$ $t \log _{2}^{1 / 2-\varepsilon}(2 / t)$.

Из этой теоремы и результатов [5] получаем

СлЕдСТВИЕ. Если $p \in[1,2)$, то не существует СП $X$, для которого

$$
\left\|\sum_{i \neq j} a_{i, j} r_{i} r_{j}\right\|_{X} \asymp\left(\sum_{i \neq j}\left|a_{i, j}\right|^{p}\right)^{1 / p} .
$$

ЗАмечАниЕ 6. В случае обычной системы Радемахера ситуация совершенно иная $[5,9]$. Так, например, в [9] показано, что для любого пространства 
последовательностей $E$, интерполяционного между $l_{1}$ и $l_{2}$, найдется функциональное СП $X$ на $I$, такое, что

$$
\left\|\sum_{j=1}^{\infty} c_{j} r_{j}\right\|_{X} \asymp\left\|\left(c_{j}\right)\right\|_{E} .
$$

Основная идея доказательств теорем 1-4 состоит в "разделении» переменных, т. е. переходе от функций вида (1) к функциям вида

$$
y(s, t)=\sum_{i \neq j} b_{i, j} r_{i}(s) r_{j}(t) \quad(s \in I, t \in I) .
$$

Для множества функций вида $(2)$ и СП $X(I \times I)$ на квадрате $I \times I$ справедливы утверждения, аналогичные приведенным.

\title{
ЛитератУРА
}

1. Качмаж C., Штейнгауз $\Gamma$. Теория ортогональных рядов. Физматгиз, М., 1958. 2. Ledoux M., Talagrand M. Probability in Banach spaces. Springer-Verlag, 1991. 3. Plichko A. M., Popov M. M. Symmetric function spaces on atomless probability spaces. Diss. Math. Warszawa, 1990. 4. Крейн С. Г., Петунин Ю. И., Семенов Е. М. Интерполяция линейных операторов. Наука, M., 1978. 5. Rodin V. A., Semyonov E. M. Anal. Math., 1, No. 3, 207-222 (1975). 6. Красносельский М. А., Рутичкий Я. Б. Выпуклые функции и пространства Орлича. Физматгиз, М., 1958. 7. Родин B. A., Ceменов E. М. Функц. анализ и его прил., 13, вып. 2, 91-92 (1979). 8. Lindenstrauss J., Tzafriri L. Classical Banach spaces 2. Springer-Verlag, Berlin, 1979. 9. Асташкин C. В. Изв. РАЕН, сер. МММИУ, 1, № 1, 18-35 (1997).

Самарский государственный университет

Поступило в редакцию 10 ноября 1997 г.

УДК 517.9

\section{Об абстрактном аналоге преобразования Крылова-Боголюбова в теории возмущенных линейных операторов*}

\author{
(c) 1999. А. Г. БАСКАКОВ
}

Пусть $\mathscr{X}$ - банахово пространство над полем $K \in\{\mathbb{R}, \mathbb{C}\}$ и $B(a, \rho)$ открытый шар в $\mathscr{X}$ с центром в точке $a \in \mathscr{X}$ и радиусом $\rho>0$. Символом $\operatorname{Lip}^{k}(\mathscr{X}, a, \rho), k \in \mathbb{N} \cup\{0\}$, обозначим банахово пространство непрерывных на замкнутом шаре $\bar{B}(a, \rho)=\overline{B(a, \rho)}$ отображений (операторов), принимаюших значения в $\mathscr{X}$ и $k$ раз дифференцируемых на $B(a, \rho)$, причем $k$-я производная удовлетворяет условию Липшица. Положим $\operatorname{Lip}(\mathscr{X}, a, \rho)=\operatorname{Lip}^{0}(\mathscr{X}, a, \rho)$.

Пусть $\mathscr{A}: D(\mathscr{A}) \subset \mathscr{X} \rightarrow \mathscr{X}$ - замкнутый линейный оператор с плотной областью определения $D(\mathscr{A})$ (ему отводится роль невозмущенного оператора). В данной заметке рассматривается возмущенное отображение вида $\mathscr{L}=\mathscr{A}-\varepsilon \mathscr{F}: D(\mathscr{A}) \cap \bar{B}(0, \rho) \subset \mathscr{X} \rightarrow \mathscr{X}$, где $\mathscr{F} \in \operatorname{Lip}^{1}(\mathscr{X}, 0, \rho), \rho>0$ и

* Работа выполнена при финансовой поддержке Российского фонда фундаментальных исследований, проект 98-01-01035. 